\title{
Effect of anti-VEGF intravitreal injection on emmetropization process
}

Keywords: retinopathy of prematurity, intravitreal bevacizumab injection, diode laser therapy, refractive error, biometry, emmetropization

\section{Opinion}

Emmetropization is a process in which, relatively wide range of refractive error in newborns, get narrower toward hyperopia in the first few years of life. The changes include vitreous cavity elongation; balanced by corneal curvature reduction and crystalline lens power reduction by getting thinner. To understand this process it is needed to assess the both refractive and biometric indices of eyes early in life, which is challenging especially in regard of biometry. Any change in this early stage of life may interfere with the emmetropization process and cause to change in refractive status of eye, amblyopia and low visual function later on life. Premature neonates are especially subject to disruption of this process. Many studies have proven that preterm neonates are more myopic than term infants. Prematurity is associated with 3 type of myopia: firstly physiological myopia that is temporary; secondly myopia without Retinopathy of Prematurity (ROP); and thirdly myopia induced by severe ROP. Many factors were speculated in this regard, including bone deficiency, temperature, light, visual deprivation and retinal dysfunction. Not only the ROP disease and its severity, but also the treatment employed believed to lead to myopia. However, there is no agreement if the treatment itself is a factor or severity of disease which necessitate treatment will lead to myopia. ${ }^{1-3}$ To isolate the effect of disease severity from treatment effect, pre- and post treatment designed studies are indicated.

Laser therapy in ROP patients resulted in significant myopia (55.2 to $80.04 \%$ ) in previous studies. ${ }^{4-6}$ It is well established that myopia associated with prematurity and conventionally (cryo- or laser therapy) treated ROP is caused by anterior segment arrest; an emmetropization disruption includes corneal steepening, anterior chamber depth reduction and lens thickening, rather than axial elongation. It has been shown also that ROP associated high myopia is primarily caused by lenticular thickening. ${ }^{7}$

Intravitreal bevacizumab (IVB) injection in ROP has shown promises in this regard and less myopia was resulted; alongside better anatomic and functional results, especially in patients with more posterior disease and zone I disease. In an unpublished review of current literature by our center which is going to be published soon, there is a mixed published result in regard of anti-VEGF effect, ranged from the same refractive outcomes as laser therapy to a less myopia degree. Mean refractive error was myopic in some $40 \%, 90 \%$ and $50 \%$ of studies with IVB monotherapy laser photocoagulation monotherapy, and IVB+ laser combination therapy, respectively. But current literature is suffering from lack of large and well matched cases as well as not harmonized inclusion criteria. In term of biometry assessment this shortage is more prominent. Few small studies have reported few biometry indices after IVB injections. ${ }^{3,8-16}$ Some authors have proposed that anti-VEGF agents minimally disrupt anterior segment development, so less myopia is expected. ${ }^{3}$ In one of our studies, we have evaluated pre- and post-treatment refractive error

\author{
Volume 4 Issue 6 - 2016 \\ Joobin Khadamy ${ }^{1,2}$ \\ 'Eye Research Center, Iran University of Medical Science(IUMS), \\ Iran \\ ${ }^{2}$ Eye Department, University of Medical Sciences (BIUMS), Iran
}

Correspondence: Joobin Khadamy, Eye research Center, Rassoul Akram hospital, Sattarkhan - Niayesh Street, Tehran |4456-13 |31, Iran, Tel +989 |25394802,

Email joobin_kh@hotmail.com Received: September 01, 2016 | Published: September 0I,
2016

and biometric indices in ROP patients treated with either laser or IVB injection monotherapy. Study has found that eyes growth in general and crystalline lens in particular is less affected by IVB treatment, in contrast to laser treatment. This supports previous proposals that anterior segment arrest which was first described for laser therapy in ROP, occurs minimally after IVB injections, if any. ${ }^{16}$ Effect of antiVEGF on ocular growth has not been fully understood.

To fully evaluate the effect of anti-VEGF treatment in eye's growth and emmetropization process, larger well designed and randomized studies with a harmonized allocation of patients for each treatment (IVB or laser treatment) are warranted. It should be also highlighted that pre- and post treatment design to measure refractive and biometric indices is necessitated to isolate the effect of treatment from severity of disease. This may help to solve longstanding discussion; if the disease severity or the treatment itself, will lead to high myopia associated with $\mathrm{ROP}^{3}$

\section{Acknowledgments}

None.

\section{Conflicts of interest}

The authors declare there are no conflicts of interest.

\section{Funding}

None.

\section{References}

1. O'Connor AR, Wilson CM, Fielder AR. Ophthalmological problems associated with preterm birth. Eye (Lond). 2007;21(10):1254-1260.

2. Hsieh CJ, Liu JW, Huang JS, et al. Refractive outcome of premature infants with or without retinopathy of prematurity at 2 years of age: A prospective controlled cohort study. Kaohsiung J Med Sci. 2012;28(4):204-211.

3. Geloneck MM, Chuang AZ, Clark WL, et al. Refractive Outcomes Following Bevacizumab Monotherapy Compared With Conventional Laser Treatment: A Randomized Clinical Trial. JAMA Ophthalmol. 2014;132(11):1327-1333. 
4. Sahni J, Subhedar NV, Clark D. Treated threshold stage 3 versus spontaneously regressed subthreshold stage 3 retinopathy of prematurity: a study of motility, refractive, and anatomical outcomes at 6 months and 36 months. Br J Ophthalmol. 2005;89:154-159.

5. Axer-Siegel R, Maharshak I, Snir M, et al. Diode laser treatment of retinopathy of prematurity: anatomical and refractive outcomes. Retina. 2008;28(6):839-846.

6. Dhawan A, Dogra M, Vinekar A, et al. Structural sequelae and refractive outcome after successful laser treatment for threshold retinopathy of prematurity. J Pediatr Ophthalmol Strabismus. 2008;45(6):356-361.

7. Garcia-Valenzuela E, Kaufman LM. High Myopia Associated With Retinopathy of Prematurity Is Primarily Lenticular. $J$ AAPOS. 2005;9(2):121-128.

8. Gunay M, Celik G, Gunay BO, et al. Evaluation of 2-year outcomes following intravitreal bevacizumab (IVB) for aggressive posterior retinopathy of prematurity. Arq Bras Oftalmol. 2015;78(5):300-304.

9. Isaac M, Mireskandari K, Tehrani N. Treatment of type 1 retinopathy of prematurity with bevacizumab versus laser. J AAPOS. 2015;19(2):140 144

10. Kuo HK, Sun IT, Chung MY, et al. Refractive Error in Patients with Retinopathy of Prematurity after Laser Photocoagulation or Bevacizumab Monotherapy. Ophthalmologica. 2015;234(4):211-217.
11. Araz-Ersan B, Kir N, Tuncer S, et al. Preliminary Anatomical and Neurodevelopmental Outcomes of Intravitreal Bevacizumab As Adjunctive Treatment for Retinopathy of Prematurity. Curr Eye Res. 2015;40(6):585-591.

12. Hwang CK, Hubbard GB, Hutchinson AK, et al. Outcomes after Intravitreal Bevacizumab versus Laser Photocoagulation for Retinopathy of Prematurity: A 5-Year Retrospective Analysis. Ophthalmology. 2015;122(5):1008-1015.

13. Chen YH, Chen SN, Lien RI, et al. Refractive errors after the use of bevacizumab for the treatment of retinopathy of prematurity: 2-year outcomes. Eye (Lond). 2014;28(9):1080-1087.

14. Harder BC, Schlichtenbrede FC, von Baltz S, et al. Intravitreal bevacizumab for retinopathy of prematurity: refractive error results. $\mathrm{Am} \mathrm{J}$ Ophthalmol. 2013;155(6):1119-1124 e1.

15. Harder BC, von Baltz S, Schlichtenbrede FC, et al. Early refractive outcome after intravitreous bevacizumab for retinopathy of prematurity. Arch Ophthalmol. 2012;130(6):800-801.

16. Reza Asadi, Mostafa Soltan-Sanjari, Joobin Khadamy, et al. Does Intravitreal Bevacizumab Injection for Retinopathy of Prematurity Treatment Arrest Anterior Segment Development? JOJ Ophthal. 2016;1:2. 